\title{
Alimentary tract innervation deficits and dysfunction in mice lacking GDNF family receptor $\alpha 2$
}

\author{
Jari Rossi, ${ }^{1}$ Karl-Heinz Herzig, ${ }^{2}$ Vootele Võikar, ${ }^{1}$ Päivi H. Hiltunen, ${ }^{1}$ Mikael Segerstråle, ${ }^{3}$ \\ and Matti S. Airaksinen ${ }^{1}$ \\ ${ }^{1}$ Program in Molecular Neurobiology, Institute of Biotechnology, University of Helsinki, Helsinki, Finland \\ ${ }^{2}$ Department of Biotechnology and Molecular Medicine, A.I. Virtanen Institute for Molecular Sciences, \\ Department of Surgery, University of Kuopio, Kuopio, Finland \\ ${ }^{3}$ Department of Animal Physiology, Viikki Biocenter, University of Helsinki, Helsinki, Finland
}

\begin{abstract}
Subsets of parasympathetic and enteric neurons require neurturin signaling via glial cell line-derived neurotrophic factor family receptor $\alpha 2$ (GFR $\alpha 2$ ) for development and target innervation. Why GFR $\alpha 2$-deficient (Gfra2 ${ }^{-/}$) mice grow poorly has remained unclear. Here, we analyzed several factors that could contribute to the growth retardation. Neurturin mRNA was localized in the gut circular muscle. GFR $\alpha 2$ protein was expressed in most substance P-containing myenteric neurons, in most intrapancreatic neurons, and in surrounding glial cells. In the $\mathrm{G} f r a 2^{-/-}$mice, density of substance $\mathrm{P}$-containing myenteric ganglion cells and nerve bundles in the myenteric ganglion cell layer was significantly reduced, and transit of test material through small intestine was $25 \%$ slower compared to wild-type mice. Importantly, the knockout mice had approximately $80 \%$ fewer intrapancreatic neurons, severely impaired cholinergic innervation of the exocrine but not the endocrine pancreas, and increased fecal fat content. Vagally mediated stimulation of pancreatic secretion by 2-deoxy-glucose in vivo was virtually abolished. Retarded growth of the $\mathrm{Gfra} 2^{-/-}$mice was accompanied by reduced fat mass and elevated basal metabolic rate. Moreover, the knockout mice drank more water than wildtype controls, and wet-mash feeding resulted in partial growth rescue. Taken together, the results suggest that the growth retardation in mice lacking GFR $\alpha 2$ is largely due to impaired salivary and pancreatic secretion and intestinal dysmotility.
\end{abstract}

J. Clin. Invest. 112:707-716 (2003). doi:10.1172/JCI200317995.

\section{Introduction}

Formation of cranial parasympathetic and enteric nervous system by neuronal precursors migrating from the neural crest requires glial cell line-derived neurotrophic factor (GDNF) signaling via GDNF family receptor $\alpha 1$ (GFR $\alpha 1)$ and Ret receptor tyrosine kinase. Loss-offunction mutations in this pathway cause Hirschsprung disease in humans and lack of cranial parasympathetic ganglia and enteric nervous system distal to the stomach in gene-knockout mice (for recent reviews see refs. 1, 2). Neurturin (NRTN) is a member of the GDNF family and signals to Ret via $\operatorname{GFR} \alpha 2(3,4)$. Cranial

Received for publication January 30, 2003, and accepted in revised form July 3, 2003

Address correspondence to: Matti S. Airaksinen, Program in Molecular Neurobiology, Institute of Biotechnology, Viikki Biocenter, 00014 University of Helsinki, Helsinki, Finland. Phone: 3589-191-59397; Fax: 3589-191-59560;

E-mail: mairaksi@operoni.helsinki.fi.

Conflict of interest: The authors have declared that no conflict of interest exists.

Nonstandard abbreviations used: glial cell line-derived neurotrophic factor (GDNF); GDNF family receptor $\alpha 1$ (GFR $\alpha 1$ ); neurturin (NRTN); substance P (SP); neuronal nitric oxide synthase (nNOS); vesicular acetylcholine transporter (VAChT); tyrosine hydroxylase (TH); vasoactive intestinal peptide (VIP); acetylcholinesterase (AchE); 2-deoxy-D-glucose (2-DG); basal metabolic rate (BMR); deep muscular plexus (dmp); postnatal day 4 (P4); megaunit (MU). parasympathetic neurons were shown to switch before birth from GDNF dependency to require NRTN for trophic support and for innervation of target tissues $(5$, 6). Mice lacking NRTN $\left(\mathrm{Nrtn}^{-/-}\right)$or GFR $\alpha 2$ (Gfra2-/-) have similar deficits in cholinergic innervation of salivary glands and small intestine $(7,8)$. In contrast to the $\mathrm{Nrtn}^{-/-}$mice (7), however, the Gfra2 ${ }^{-/-}$mice grow poorly after weaning (8), but the mechanisms of this growth impairment have remained unclear.

We hypothesized that the apparent malnutrition in the Gfra $2^{-/-}$mice could be due to functional innervation deficits in the gastrointestinal tract and/or in feeding control. In this study, we first analyzed the cellular subtype markers colocalized with GFR $\alpha 2$ protein in the gut and pancreas. Second, we compared substance $\mathrm{P}$-containing innervation in the small intestine and the in vivo motility between wild-type and Gfra2 $2^{-/-}$mice. Third, because parasympathetic neurons mediate the food-induced pancreatic exocrine secretion via a vagal pathway $(9,10)$, we investigated possible deficits in the intrinsic innervation of the exocrine pancreas in the Gfra $2^{-/-}$mice and their functional consequences in vivo. Furthermore, we monitored factors that could contribute to differences in growth, such as food and water consumption, metabolic rate, and motor activity. Finally, given the innervation deficit of the salivary gland, we examined if the impaired growth of the Gfra2-/- mice could be rescued by wet-mash feeding. 


\section{Methods}

Animals. Gfra2 ${ }^{-/}$and wild-type littermates in (C57BL/6 $\times 129 \mathrm{~Sv}) \mathrm{F}_{1}$ hybrid background were obtained by mating congenic GFR $\alpha 2$ heterozygous mice. The congenic mice were produced by backcrossing the heterozygote mice separately with wild-type mice of the two background strains (C57BL/6JOlaHsd and 129SvHsd) for at least nine generations. The animals were genotyped as described previously (8). The mice were kept in standard, specific pathogen-free conditions under a constant dark/light cycle. All animal experiments were approved by the local ethical committee for animal research at the University of Helsinki.

Histology. Nrtn mRNA in situ hybridization on cryosections from mouse intestines was performed as described (8). No specific labeling above background was seen in an adjacent section hybridized with a sense probe. For immunohistochemistry, mice were anesthetized with chloral hydrate and perfused transcardially with $4 \%$ paraformaldehyde in PBS ( $\mathrm{pH}$ 7.5). The gut and pancreas were carefully removed. For whole-mount staining, gut samples were prepared from duodenum (immediately caudally to antrum) and from ileum (cranially to ileocecal junction). To visualize substance $\mathrm{P}$-containing (SP-containing) myenteric ganglion cells, some samples were cultured for 12 hours in colchicine-containing medium as described (11). Samples were opened along their mesenteric border, and the muscular layers were separated from the mucosal layer. Depending on the $\mathrm{Ab}$ used, the samples were stained immediately or fixed overnight at $4^{\circ} \mathrm{C}$. Cryostat sections and whole-mount preparations were stained using standard immunofluorescence techniques. Primary Ab's against GFR $\alpha 2$ were as follows: (goat polyclonal $\mathrm{Ab}$ ) from R\&D Systems Inc. (Minneapolis, Minnesota, USA); neuronal nitric oxide synthase (nNOS; rabbit polyclonal Ab); PGP9.5 (rabbit polyclonal $\mathrm{Ab}$ ); vesicular acetylcholine transporter (VAChT; goat polyclonal $\mathrm{Ab}$ ) and tyrosine hydroxylase $(\mathrm{TH}$; sheep polyclonal $\mathrm{Ab}$ ) from Chemicon International (Temecula, California, USA); $S 100 \beta$ (rabbit polyclonal $\mathrm{Ab}$ ) from SWant (Bellinzona, Switzerland); SP (rat mAb) from Medicorp (Montreal, Quebec, Canada); and vasoactive intestinal peptide (VIP; rabbit polyclonal) from Progen Biotechnik GmbH (Heidelberg, Germany). Fluorescent cyanine (Cy2)- or indocarbocyanine (Cy3)-labeled donkey secondary Ab's were from Jackson ImmunoResearch Laboratories Inc., West Grove, Pennsylvania, USA). NADPH-diaphorase and acetylcholinesterase (AChE) histochemistry were performed as described previously (8). Samples were viewed under a microscope fitted with digital camera and image-processing program (Carl Zeiss Microscopy, Jena, Germany). For colocalization, a $\times 63$ oil objective lens was used. For some samples, confocal $0.5-\mu \mathrm{m}$ optical sections were generated with a Bio-Rad Laboratories Inc. (Hercules, California, USA) imaging system equipped with appropriate lasers and software.

The density of SP-positive fibers in the whole mounts was quantified with $\times 40$ magnification by counting fibers crossing a standardized grid from five positions per sample. The density of SP-positive somas in the whole mounts was quantified with $\times 20$ magnification from randomly selected fields throughout the duodenum. For adult intrapancreatic neurons, NADPH diaphorase-labeled cell profiles containing a clear, unlabeled nucleus were counted from every third through fifth section throughout all regions of the pancreas. PGP9.5-labeled intrapancreatic neuronal profiles were counted from every fourth through eighth section through the whole pancreas from 4-day-old mice. The average number of labeled profiles per ganglion (single profiles and grouped together) and the number of labeled ganglia per animal was calculated. The density of AChE- and TH-positive fibers in pancreas was quantified with the Adobe Photoshop program as the number of positive pixels in image field (eight images per animal taken at two section levels and from four randomly selected positions per section covering all regions of the exocrine pancreas).

In vivo motility. The charcoal transit test method was modified as described (12). Mice (2- to 3-month-old littermates of both sex) fasted overnight were administered a bolus of $100 \mu \mathrm{l}$ charcoal-water mixture (Carbomix; Oy Leiras Finland Ab, Turku, Finland) into the stomach by orogastric gavage. The animals were sacrificed after 90 minutes, the intestine was frozen in icecold PBS and straightened on a flat surface, and the distance traveled by the bolus was measured. Results were expressed as the ratio (percentage) of the distance traveled by the bolus to the total length of the small intestine (pylorus-cecum). The average length of small intestine was identical between the genotypes.

Analysis of pancreatic secretion in vivo. The method used for in vivo collection of bile and pancreatic secretion was modified as described previously (13). Briefly, mice (3- to 5-month-old mice of both gender) fasted overnight were anesthetized with urethane $(1.3 \mathrm{~g} / \mathrm{kg}$ body weight; Sigma-Aldrich, St. Louis, Missouri, USA). Body temperature was maintained on a heating pad and the duodenum and pancreas were exposed through a midline abdominal incision. The common bile-pancreatic duct was cannulated at the sphincter of Oddi with a polyethylene catheter $(0.28 \mathrm{~mm}$ inside diameter $\times 0.61 \mathrm{~mm}$ outside diameter). After collecting the basal secretion for 60 minutes at 15 -minute intervals, 2-deoxy-D-glucose (2-DG; $6 \mathrm{mg} / \mathrm{kg}$, diluted in $0.9 \%$ saline) was injected (in $0.1 \mathrm{ml}$ volume) into the peritoneal cavity. The incision was covered with moistened gauze. The diverted bile-pancreatic juice was collected for up to 120 minutes in previously weighed tubes at 15-minute intervals, and the volumes were determined. Protein concentration was measured using a Bradford assay (Bio-Rad Laboratories Inc.). Amylase activity was measured using 2-chloro-4-nitrophenyl- $\alpha$-D-maltotrioside as substrate (14). For each animal, results were expressed as the percentage of change in protein or amylase secretion (per 15 minutes) from the animal's average prestimulus secretion. 

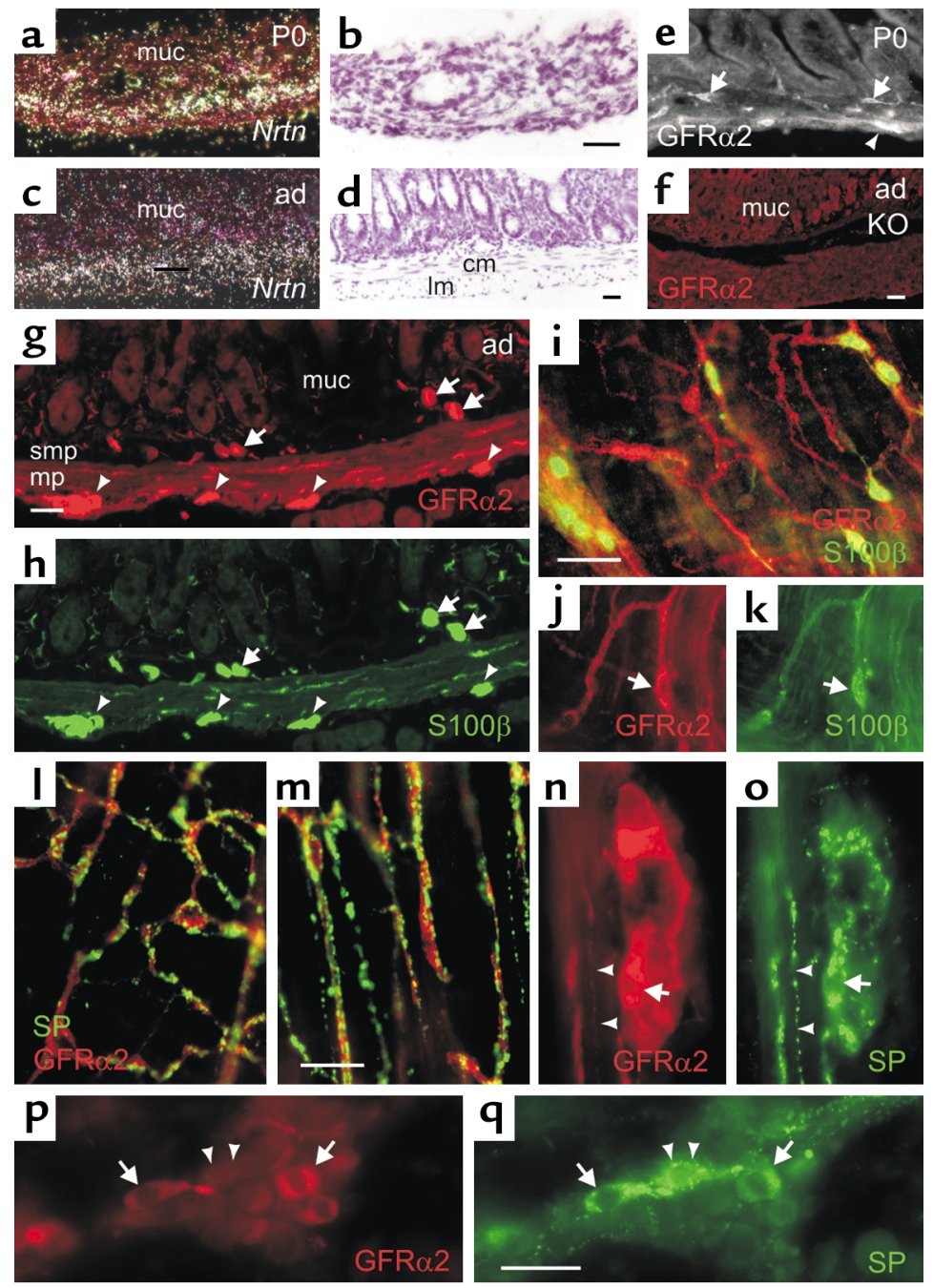

\section{Figure 1}

Expression of Nrtn mRNA and GFR $\alpha 2$ protein in alimentary tract. (a-d) Localization of Nrtn mRNA in mouse gut by in situ hybridization. ( $\mathbf{b}$ and $\mathbf{d}$ ) Corresponding brightfield images of dark-field images a and c, respectively. Nrtn is strongly expressed in the circular muscle layer at birth ( $\mathbf{a}$ and $\mathbf{b}$ ), but its levels decrease in adults ( $\mathbf{c}$ and $\mathbf{d}$ ). Distribution of GFR $\alpha 2$ protein in a cross section of the small intestine from newborn (e) and adult ( $\mathbf{g}$ and $\mathbf{n}$ ) wild-type mouse. No specific labeling is seen in a corresponding section from adult Gfra $2^{-/-}$mice (f). (g) GFR $\alpha 2$ is found in myenteric (arrowheads) and to submucosal ganglia (arrows). (h) Immunostaining for $\mathrm{S} 100 \beta$ shows that glial cells in the muscle and mucosa are also labeled. (i-m) Whole-mount preparation of duodenum from adult wild-type mouse immunostained for GFR $\alpha 2$ (red) and $\mathrm{S} 100 \beta$ (i and $\mathbf{k}$, green) or SP ( $\mathbf{I}$ and $\mathbf{m}$, green), focused at the same level as the myenteric ganglion cells (i-I) or deep muscular plexus (m). (i-k) S100 $\beta$-positive enteric glia (arrows) express GFR $\alpha 2$. (I and $\mathbf{m}$ ) SP-containing secondary and tertiary nerve fiber bundles are GFR $\alpha 2$ positive. (n-q) Myenteric ganglia stained for GFR $\alpha 2$ ( $\mathbf{n}$ and $\mathbf{p}$ ) and SP (o and $\mathbf{q}$ ) in a cross section ( $\mathbf{n}$ and $\mathbf{o}$ ) or in colchicine-treated whole-mount preparation ( $\mathbf{p}$ and $\mathbf{q})$. Arrowheads point at SP-positive, GFR $\alpha 2$-negative structures. Arrows point at doublelabeled neurons. Bars in $\mathbf{a}-\mathbf{h}=100 \mu \mathrm{m} ; \mathbf{i}-\mathbf{q}=50 \mu \mathrm{m}$. $\mathrm{cm}$, circular muscle; Im, longitudinal muscle layer; muc, mucosa; ad, adult; $\mathrm{P} 0$, postnatal day $0 ; \mathrm{mp}$, myenteric plexus; smp, submucosal plexus.
Fecal-fat analysis. Fecal fat was extracted and measured as described (15) from stool samples collected from 4week-old female mice kept for 1 week on a high-fat diet (Diet 1060; Altromin, Lage, Germany), 40\% calories from fat). Briefly, total fecal lipids were extracted from dried fecal samples after homogenization first with heptane/diethylether/ethanol (1:1:1 vol/vol) and then twice with heptane/diethylether/ethanol/water (1:1:1:1 $\mathrm{vol} / \mathrm{vol})$. Supernatants from each extraction were collected in previously weighed glass vials, and lipid content was measured gravimetrically after solvent evaporation. Total fecal lipid amount was expressed as a percentage of the weight of the starting fecal sample.

Food and water intake. Four-month-old animals were singly housed in standard plastic cages with a stainless steel grid on the bottom and filter paper below the grid. Standard dry food (1320 breeding diet; Altromin) was administered in stainless steel cups (Plexx, Elst, The Netherlands) and water administered in $15-\mathrm{ml}$ plastic tubes. Mice were habituated with this unfamiliar regime for 2 days, after which the food and water intake was measured for 5 days. The animals and the amount of food and water consumed were weighed daily at 11:00 a.m. At the same time, the food cups and bottles were removed, the cage was cleaned, and all fecal boli and spilled food were collected from the bottom. Thereafter, the mice were provided with fresh food and water. The food-intake results were expressed as daily food intake per (body weight $)^{0.75}$.

Metabolic rate. To determine the basal metabolic rate (BMR) we used open-flow respirometry measuring the produced $\mathrm{CO}_{2}$ with an infrared $\mathrm{CO}_{2}$ sensor (Qubit Systems, Ottawa, Ontario, Canada). The animals were fasted overnight, weighed, and placed individually in Plexiglas chambers 2 hours prior to the experiment at $25^{\circ} \mathrm{C} \pm 1{ }^{\circ} \mathrm{C}$. Dried room air was pumped through at a rate of $400 \mathrm{ml} / \mathrm{min}$, and reference values for $\mathrm{CO}_{2}$ were taken immediately before the measurements. The produced $\mathrm{CO}_{2}$ was measured at $1 \mathrm{~Hz}$ for 1 hour, and the average of the lowest 5-minute plateau values was considered as BMR for each individual animal (see, for example, ref. 16).

Motor activity. Three- to five-month-old mice $(n=10$ in both genotypes) were placed singly in transparent plastic cages that had food and fresh sawdust spread on the bottom. The cages were placed in activity monitors 
(MED Associates Inc., St. Albans, Vermont, USA), and monitoring began at 11:00 a.m. Infrared photo-beam interruptions were registered and analyzed by the software of the activity monitor. The activity was monitored continuously over 24 hours and expressed as distance traveled in 30-minute time periods.

Wet-mash feeding. Male mice from several litters derived from $\mathrm{F}_{1}$ homozygous matings were used for growth measurements in each feeding group. The mice were given either dry pellets (1320 diet; Altromin) or a wet-mash mixture (2:1) made up of ground dry pellets and water (wet-mash diet). Fresh wet-mash meal was given daily starting 1 week before the weaning (at around 3 weeks of age). Growth was followed by weekly weight measurements (from 19 up to 61 days of age). Drinking water was offered ad libitum.

Statistical analyses. Results are expressed as mean plus or minus SEM. ANOVA was used to compare the two genotypes in experiments on circadian activity and food intake. The data from all other tests were compared using a two-tailed Student $t$ test, assuming unequal variance. $P$ values of less than 0.05 were considered to be statistically different.

\section{Results}

The growth of Gfra2 $2^{--}$mice derived from homozygous or heterozygous matings did not differ (not shown), and their body weights were significantly lower compared with the wild-type controls, as described earlier (8). Histological assessment by $\mathrm{H} \& \mathrm{E}$ staining did not reveal morphological differences in the salivary glands, bowel, or pancreas.

Expression of Nrtn mRNA in gut muscle and GFR $\alpha 2$ protein in enteric neurons and glia. In postnatal mouse intestine, mRNA expression of GFR $\alpha 2$ ligand (Nrtn) was predominantly seen in the circular muscle layer of newborns (Figure 1, a and b) and adults (Figure 1, $c$ and d). GFR $\alpha 2$ protein was expressed in myenteric and submucous nerve plexuses and ganglia in newborn (Figure 1e) and adult (Figure 1, g-o) mouse small intestines. Specificity of the labeling was confirmed by using sections from Gfra $2^{-/-}$mice (Figure 1f). S100ß-labeled enteric glia within the ganglia and in muscle and mucosal layers were GFR $\alpha 2$ immunoreactive (Figure 1, $\mathrm{g}-\mathrm{k})$. Double-staining of SP and GFR $\alpha 2$ in the myenteric plexus of small intestine showed a similar pattern of labeling (Figure 1,1-o) in fiber bundles at the level of the myenteric ganglion cells (Figure 1, g and $\mathrm{l}$ ) and deep muscular plexus (dmp, Figure $1 \mathrm{~m}$ ), a layer near the boundary of the innermost circular muscle with the submucosa (17). In the myenteric ganglia, SP and GFR $\alpha 2$ were mostly colocalized (Figure $1, n-q)$. Of 200 labeled cells counted, a subset of the SP-positive cells was GFR $\alpha 2$ negative (39 of 162) and a subset of GFR $\alpha 2$-positive cells was SP negative (38 of 161).

Decreased SP innervation of small bowel and impaired in vivo motility in GFR $\alpha 2$-deficient mice. We have shown previously that the density of AChE-positive fiber network is reduced in the small intestine of $\mathrm{Gfra}^{-/-}$mice, sug- gesting a deficit in the excitatory innervation of gut muscles (8). Whole-mount immunostaining of small bowel muscle preparations revealed a significantly reduced density of SP-positive nerve fiber bundles in the myenteric ganglion cell layer in Gfra2 ${ }^{-/-}$mice compared with wild-type animals (duodenum: KO, $0.11 \pm 0.01$, versus WT, $0.43 \pm 0.03$; ileum: $\mathrm{KO}, 0.16 \pm 0.02$, versus WT, $0.43 \pm 0.03 ; P<0.001, n=3$ in each group). The reduction was most pronounced in the tertiary plexus that forms a network of thin-caliber fiber bundles in the spaces between the primary plexus (Figure 2, a and b). The density of nerve fibers double-labeled for SP and a general neuronal marker PGP9.5 was clearly reduced in tertiary plexus in Gfra2 $-/$ - mice supporting the idea that fibers, and not only the transmitter, are reduced (not shown). In contrast, the density of SP-positive fiber bundles in the dmp layer was not significantly reduced in Gfra $2^{-/}$mice compared with wild-type animals (duodenum: KO, $0.45 \pm 0.03$, versus WT, $0.55 \pm 0.05, P=0.09$; ileum: KO, $0.48 \pm 0.03$, versus WT, $0.47 \pm 0.04 ; P=0.83$, $n=3$ in each group) (Figure 2, $\mathrm{c}$ and d).

We prepared colchicine-treated myenteric plexus whole-mounts (11) from duodenum to quantify somas expressing SP. The density of SP-containing somas was significantly reduced (about 35\%) in the knockouts compared with wild-type mice $(\mathrm{KO}, 19 \pm 2$, versus $\mathrm{WT}$, $29 \pm 1 ; P=0.02, n=3$ in both groups).

To study whether the enteric innervation deficits found in the Gfra $2^{-/-}$mice are reflected by changes in bowel motility in vivo, we examined intestinal propulsion by a charcoal transit test. In wild-type mice, intragastrically delivered test material traveled almost to the end of the small intestine during the
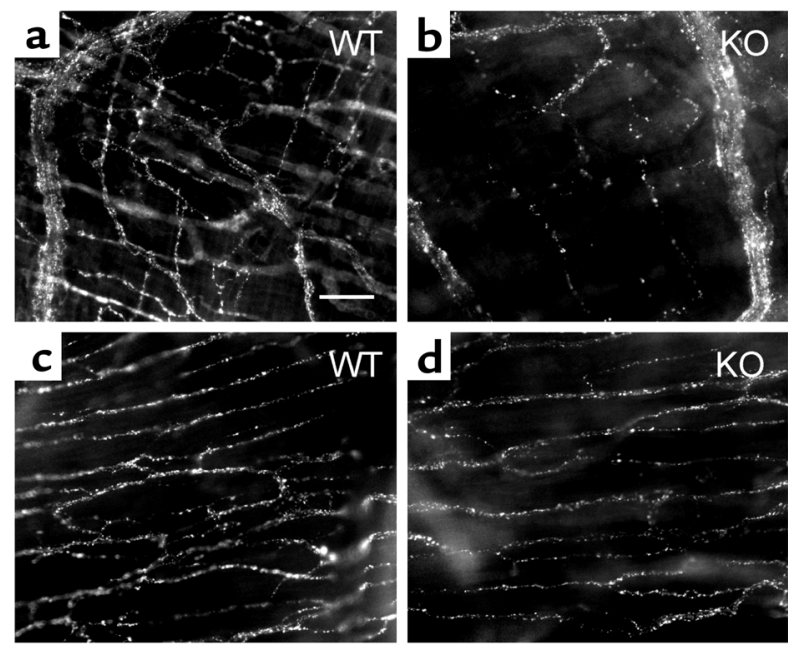

\section{Figure 2}

Deficient fine SP-positive fibers in the duodenum of Gfra $2^{-/-}$mice. (a and $\mathbf{b}$ ) In adult wild-type mice, in the same focal plane as the myenteric ganglion cells, SP-positive fiber bundles form a network in the spaces between the meshes of the primary plexus (a), whereas in Gfra $2^{-/}$(KO) mice, the density of these fiber bundles is severely reduced (b). (c and d) Density of SP-positive fibers in the deep muscular plexus at the innermost level of the circular muscle is comparable between wild type (c) and Gfra $2^{-/-}$duodenum (d). Bar, $50 \mu \mathrm{m}$. 

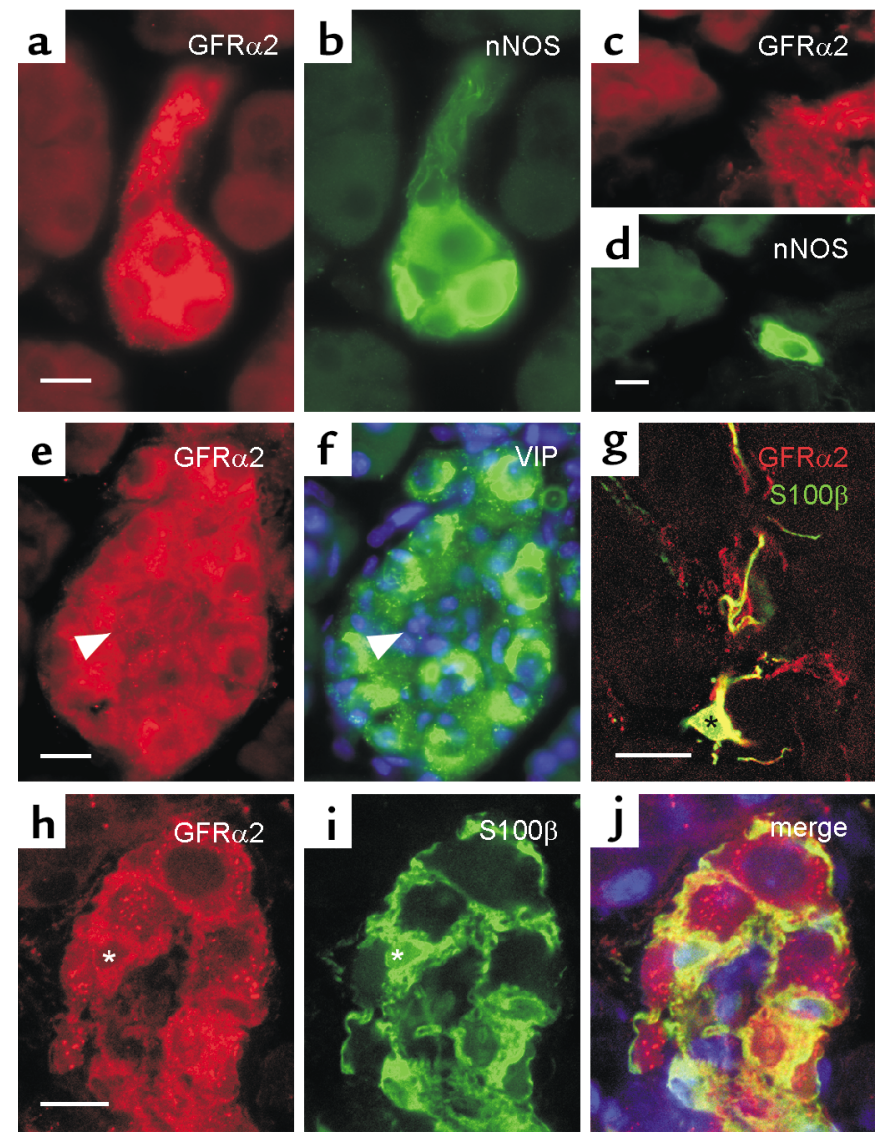

\section{Figure 3}

Localization of GFR $\alpha 2$ protein in the pancreas. (a and $\mathbf{b}$ ) An intrapancreatic ganglion, in which all the neurons are positive for GFR $\alpha 2$ (red) and nNOS (green). (c and d) A single nNOSpositive neuron in the pancreas negative for GFR $\alpha 2$. (e and $\mathbf{f}$ ) A large intrapancreatic ganglion, in which all VIP-positive (green) neurons express GFR $\alpha 2$ (red). Arrowheads mark VIP-negative, GFR $\alpha 2$-expressing cells that may be glial cells. DAPI-stained nuclei are blue. (g) Confocal optical section image showing colocalization of GFR $\alpha 2$ (red) and S100 $\beta$ (green) in exocrine pancreas. Black asterisk marks a terminal Schwann cell soma. GFR $\alpha 2$ expression is also seen in S100 $\beta$-negative nerve fibers. (h-j) Confocal optical section image through an intrapancreatic ganglion showing the colocalization of GFR $\alpha 2$ (red) and $\mathrm{S} 100 \beta$ (green) in satellite cells. A satellite cell nucleus is marked by white asterisks. DAPI-stained cell nuclei are blue in the merged image. Bars, $10 \mu \mathrm{m}$.

and c) consistent with previous data (19). In contrast, virtually no intrapancreatic ganglia (containing more than one labeled neuron) were seen in the knockouts. Only occasionally, single labeled neurons were found in some sections (Figure 4, b and d). The total number of labeled pancreatic neuronal profiles was significantly reduced $(83 \%)$ in adult Gfra $2^{-/}$mice as compared with the wild-type littermates $(\mathrm{KO}, 193 \pm 21$, versus WT, $1,075 \pm 136$; $P<0.005, n=3$ in both groups).

Using Ab's to PGP9.5 to label the intrapancreatic neurons, we found a profound $(-85 \%)$ reduction in

90-minute measuring period, whereas in Gfra $2^{-/-}$animals the distance traveled was significantly (about $25 \%$ ) shorter (percentage of the total length of the small intestine: WT, $93 \% \pm 3 \%$, versus $\mathrm{KO}, 69 \% \pm 5 \%$; $P=0.001, n=9$ in both groups).

Lack of intrapancreatic ganglion neurons and innervation of exocrine pancreas. In the adult pancreas, GFR $\alpha 2$ protein was expressed in the intrapancreatic neurons labeled for nNOS (Figure 3, a and b), VIP (Figure 3, e and f), or general neuronal marker PGP9.5 (not shown). A few (14 out of 96 counted) NOS-positive neurons, mostly found as single cells, were GFR $\alpha 2$ negative (Figure 3, $\mathrm{c}$ and d). GFR $\alpha 2$ was also expressed in $S 100 \beta$-positive satellite cells surrounding the neuronal cell bodies in intrapancreatic ganglia and in terminal Schwann cells on the axons in exocrine pancreas (Figure $3, \mathrm{~g}-\mathrm{j}$ ). Cells in intrapancreatic ganglia were GFR $\alpha 2$ positive also in newborn mice (not shown).

NRTN and GFR $\alpha 2$ are required for parasympathetic innervation of cranial exocrine glands $(5,6)$, suggesting that this signaling could be required for the innervation of other exocrine tissues including the pancreas. Consistent with this idea, Nrtn mRNA expression in the late embryonic mouse pancreas increases before birth (18). To test this hypothesis, we quantified intrapancreatic parasympathetic neurons using NADPH-diaphorase histochemistry. In wild-type mice, numerous intrapancreatic ganglia were located near the ducts consisting usually of clusters of 10-20 labeled profiles (Figure 4, a labeled pancreatic neuronal profiles in the knockout animals already at postnatal day 4 (P4) (KO, $130 \pm 40$, versus WT, $980 \pm 100$ labeled profiles per animal, $n=2$ in both groups). The average number of labeled profiles grouped together was 2.6 (range 1-13) in the wild-type and 1.6 (range 1-6) in the knockout pancreas. The number of intrapancreatic ganglia (clusters containing at least two labeled profiles) was reduced about $80 \%$ in the P4 knockout mice.

In addition, as visualized by $\mathrm{AChE}$ histochemistry, the density of cholinergic innervation in the exocrine pancreas was severely reduced (approximately 90\%; $P<0.005, n=4$ in both groups) in adult Gfra $2^{-/-}$as compared with the wild-type mice (Figure 4, e and f). In contrast with the exocrine part, islets contained cholinergic fibers in Gfra $2^{-/-}$mice (Figure 4, e and f). Similar results were obtained using other cholinergic markers VAChT and VIP (not shown). In contrast to the cholinergic innervation, the density of sympathetic innervation in the exocrine pancreas was not significantly different $(P=0.55, n=4)$ between the genotypes (Figure 4, $g$ and $h$ ).

Impaired vagally stimulated secretion of pancreatic enzymes and fat malabsorption in vivo. Parasympathetic cholinergic innervation is thought to mediate the physiological food-induced secretion of pancreatic enzymes via an afferent-efferent vago-vagal pathway $(9,10)$; therefore, we studied the consequences of reduced pancreatic innervation in the Gfra $2^{-/-}$mice upon bile-pancreatic 


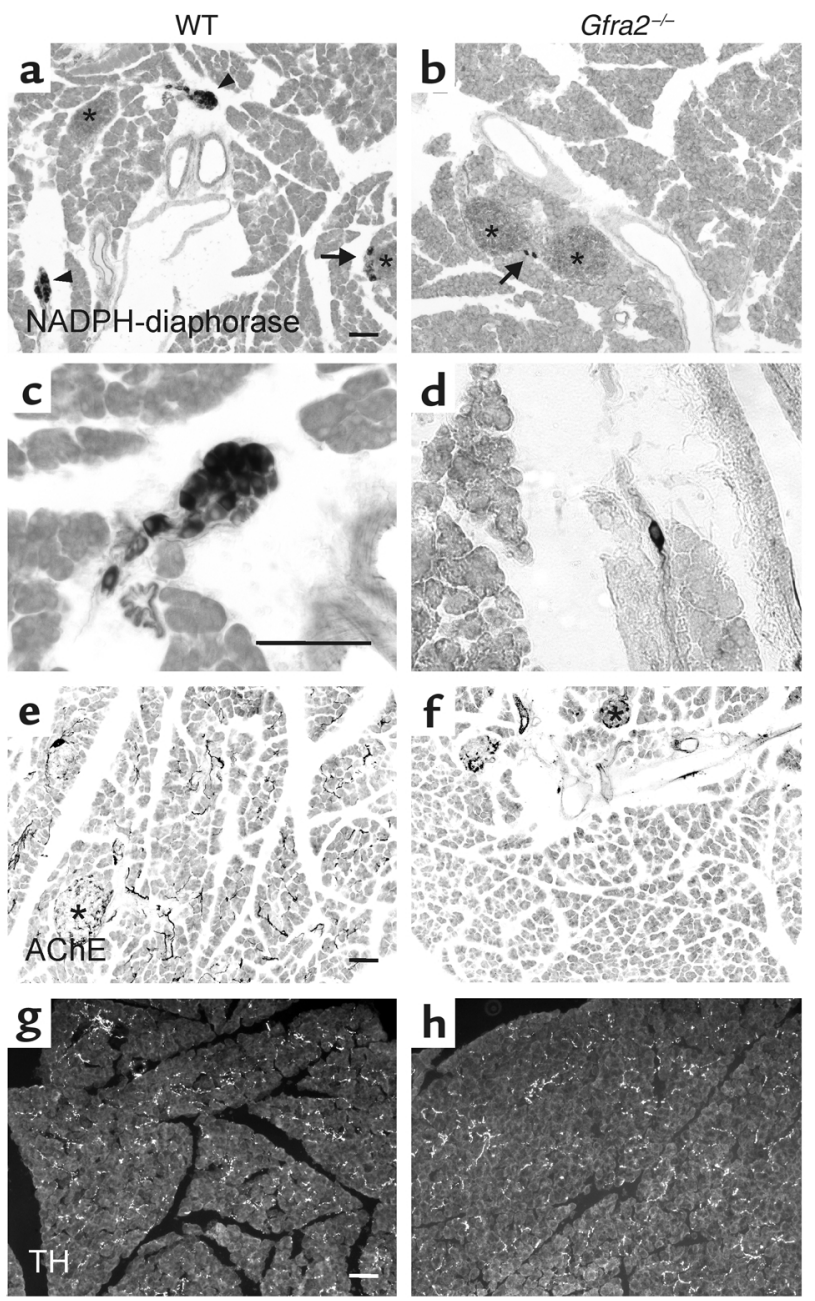

secretion in vivo using a well-known centrally acting vagal stimulant, 2 -DG $(20,21)$. Before stimulation, there was no significant difference between the wildtype $(n=10)$ and $G$ fra $2^{--}(n=8)$ mice in the fluid, protein (not shown), or amylase (WT, $34 \pm 7$ megaunits $[\mathrm{MU}] / 15 \mathrm{~min}$, versus KO, $54 \pm 13 \mathrm{MU} / 15 \mathrm{~min}, P=0.2$ ) secretion. In nonstimulated animals observed for up to 3 hours, the basal protein (not shown) and amylase (Figure 5) output decreased slightly and similarly in both genotypes. The 2-DG did not significantly change

\section{Figure 4}

Profound loss of intrapancreatic neurons and cholinergic innervation in Gfra $2^{-/-}$mouse pancreas. (a) In wild-type animals, intrapancreatic neurons labeled by NADPH-diaphorase histochemistry are seen in numerous ganglia throughout the exocrine pancreas (arrowheads). Single neurons (arrow) were observed also in the vicinity of islets of Langerhans (asterisks). (b) No intrapancreatic ganglia are found in $\mathrm{Gfra}^{-/-}$mice. The remaining few labeled neurons (arrow) are mainly located near the islets (asterisks). (c) A higher magnification of a typical ganglion in wild-type pancreas that has more than ten labeled neurons. (d) A rare example of a neuron in the exocrine part of pancreas in Gfra2 $2^{-/}$mice. (e) In wild-type animals, AChE-positive cholinergic nerve fibers are seen as scattered fiber bundles. (f) There is a profound loss of cholinergic innervation in the $\mathrm{G} f \mathrm{fr} 2^{-/-}$pancreas. Note that the cholinergic innervation of islets (asterisks) is less affected compared with the exocrine part. ( $\mathbf{g}$ and $\mathbf{h}$ ) Sympathetic innervation of $\mathrm{G}$ fra $2^{-/-}$mouse pancreas is unchanged compared with wild-type mouse pancreas. TH, tyrosine hydroxylase. Bars, $100 \mu \mathrm{m}$.

the flow of bile-pancreatic fluid secretion in the wildtype or Gfra2 $2^{--}$mice (not shown). As expected, administration of 2-DG significantly increased amylase output in the wild-type mice, whereas in the Gfra $2^{-/-}$mice there was no significant increase of amylase secretion in response to 2-DG stimulation (Figure 5). The difference in the integrated 2-DG-stimulated amylase secretion between the genotypes was significant $(P=0.01)$. Protein secretion response to 2-DG stimulation paralleled the amylase response, with a significant increase in the wild-type but not in the knockout animals (not shown).

To address the question whether the mice were malabsorbing fat in vivo, we measured stool fat on a highfat diet. This analysis indicated a significantly elevated fat content in the stool of $\mathrm{Gfra}^{-/-}$mice compared with wild-type animals (KO, $19.6 \% \pm 0.6 \%$, versus WT, $16.5 \%$ $\pm 0.3 \% ; P=0.001, n=8$ mice of both groups).

Increased food and water consumption and metabolic rate and decreased body fat. To investigate possible differences in food intake, we carried out a battery of behavioral tests using 4-month-old mice, in which the growth rate does not differ between the genotypes. Thus, the weight difference remained similar throughout the experiment. The Gfra $2^{-/-}$mice consumed slightly $(\sim 8 \%)$ but significantly more food per unit of body mass $(P<0.05$,

\section{Figure 5}

Reduced 2-DG-stimulated pancreatic secretion in $\mathrm{Gfra}^{-/-}$mice in vivo. The 2-DG, a central vagal stimulant, induces amylase secretion from wild-type mouse pancreas (filled squares). In Gfra $2^{-/-}$(KO) mice, 2-DG-stimulated amylase secretion is virtually absent (filled circles). Nonstimulated, basal pancreatic secretion does not change significantly during the 60-minute period (open symbols). The number of animals in each group is indicated in parentheses. The integrated secretion response to 2-DG stimulation (area between stimulated and basal secretion curves) was significantly different between the genotypes $(P=0.01)$. Average amylase secretion before stimulus was set as $100 \%$. For WT this represents $34 \pm 7 \mathrm{MU} / 15 \mathrm{~min}$; for KO $54 \pm 13 \mathrm{MU} / 15 \mathrm{~min}$.

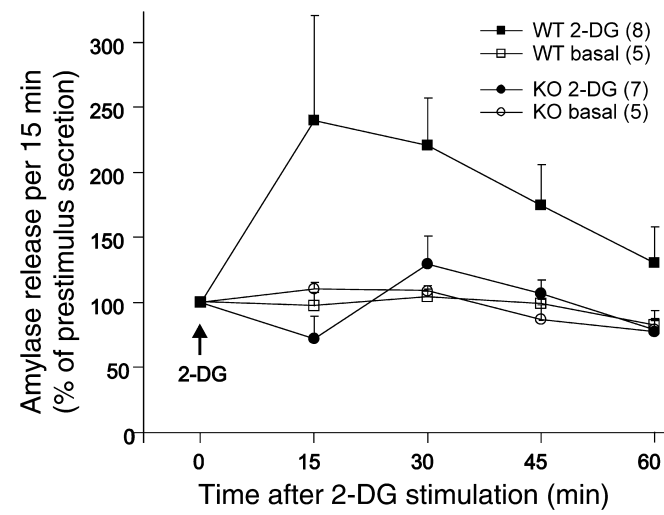



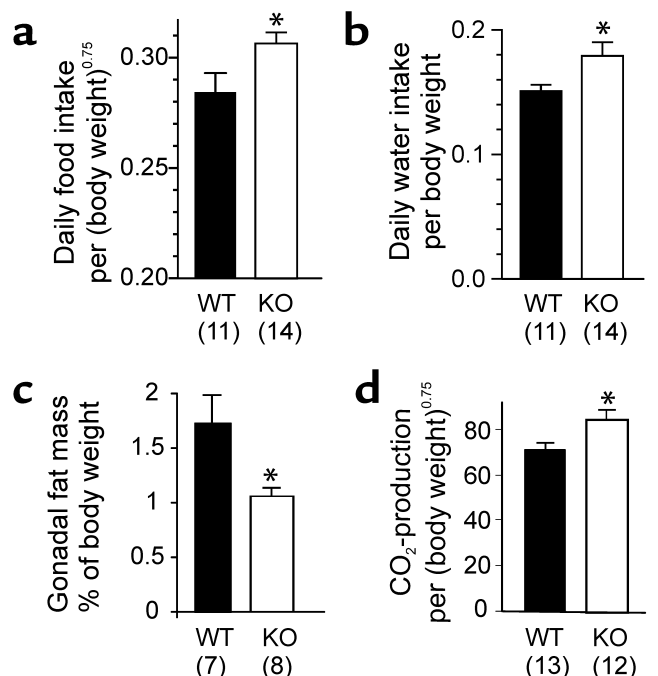

Figure 6

Food and water intake, gonadal fat mass, and $\mathrm{BMR}\left(\mathrm{CO}_{2}\right.$ production) of adult wild-type and Gfra $2^{-/-}(\mathrm{KO})$ mice under standard drypellet feeding. Because average weights differ between the genotypes, food intake and $\mathrm{CO}_{2}$ production values are standardized to (body weight $)^{0.75}$ (38). (a) Total food intake, expressed as daily intake in grams per (body weight in grams) $)^{0.75}$, is significantly increased. (b) Water intake per body weight is also increased. (c) Gfra $2^{-/-}$mice have less proportional gonadal fat compared with wild-type mice. Fat weights were measured from 4- to 6-month-old female mice. (d) The BMR, expressed as $\mathrm{CO}_{2}$ production using the equation $\left(\mu \mathrm{l} \mathrm{min}{ }^{-1}\right)$ per (body weight in grams) ${ }^{0.75}$, is significantly higher in the knockout than in the wild-type mice. The number of mice used in each experiment is shown in parenthesis below the bars. ${ }^{*} P<0.05$.

Figure 6a). Water intake per body weight was also significantly increased $(20 \%)$ in the knockout mice under dry-pellet feeding $(P<0.05$, Figure $6 \mathrm{~b})$.

We have shown previously that serum albumin levels are reduced $15 \%$ in $\mathrm{Gfra}^{-/-}$mice (8). In contrast, basal glucose levels in freely fed animals were normal (not shown). As an independent measure of nutritional state, proportional weights of gonadal fat pads as an estimate of body fat (22) were measured from 4- to 6month-old female mice (Figure 6c). The average weight of gonadal fat was $40 \%$ lower in $\mathrm{Gfra}^{-/-}$mice than in wild-type controls $(P<0.05)$. Interestingly, weight-specific $\mathrm{BMR}$ (measured from $\mathrm{CO}_{2}$ production at rest) was significantly increased $(P<0.05$, Figure 6d). Rectal temperature (measured at 11:00 a.m.) was not significantly different between the genotypes (WT, $\left.36.9 \pm 0.3^{\circ} \mathrm{C} ; \mathrm{KO}, 36.5 \pm 0.4^{\circ} \mathrm{C} ; P=0.2\right)$. Locomotor activity measurement over 24 hours (data not shown) revealed no difference between knockout $(n=10)$ and wild-type $(n=10)$ mice $(P>0.2)$. Activity was significantly increased during the dark period $(P<0.001)$ similarly in both groups $(P>0.9)$.

Partially rescued growth of $\mathrm{G} f r a 2^{-/-}$mice with wet-mash feeding. The growth of $\mathrm{Gfra2}^{-/-}$mice compared with their wild-type littermates shows a marked decrease after weaning (8). Since the Gfra2 ${ }^{-/-}$mice have a deficient innervation of salivary glands $(5,8)$ and exocrine pancreas, we compared the postweaning growth of several litters of $\mathrm{Gfra2} 2^{-/-}$mice supplied with dry- and wetmash diets. Wet-mash feeding was able to significantly enhance the growth of $\mathrm{Gfra2} 2^{--}$animals during the 61day observation period as compared with $\mathrm{Gfra}^{-/-}$mice maintained with dry food (Figure 7).

\section{Discussion}

In this study, we have investigated different aspects of alimentary tract structure and function that could contribute to the growth failure of $\mathrm{Gfra}^{-/-}$mice. The main structural findings include reduction of SP-containing nerve fibers in the myenteric plexus of the small intestine and profound loss of intrapancreatic neurons and cholinergic innervation of exocrine pancreas. Correspondingly, slower gut motility and drastically impaired vagally stimulated pancreatic secretion were observed in vivo. Moreover, Gfra $2^{-/-}$mice have elevated fecal fat and reduced body fat content, higher BMR, but normal locomotor activity. The mice also drink more water, and their growth is partially rescued by wet-mash feeding. Expression of GFR $\alpha 2$ in enteric and parasympathetic neurons and in associated glial cells. We found GFR $\alpha 2$ protein in many enteric and intrapancreatic parasympathetic ganglia in newborn and adult mice. GFR $\alpha 2$ protein was colocalized with the excitatory transmitter SP-containing nerve fibers in the myenteric plexus. In the intrapancreatic ganglia, we could quantify GFR $\alpha 2$ colocalization with different neuronal markers. GFR $\alpha 2$ was expressed in most VIP- and nNOS-positive neurons. A subpopulation ( $15 \%)$ of nNOS neurons did not express GFR $\alpha 2$ and would thus be expected to remain unaffected by lack of GFR $\alpha 2$.

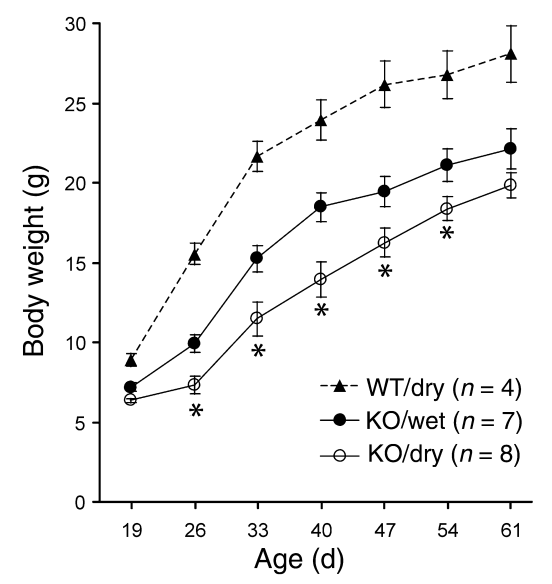

Figure 7

Partial rescue of the Gfra2-/- (KO) mice growth by wet-mash feeding. Growth curves of Gfra $2^{-/-}$male mice fed with dry- (open circles) or wet-mash (filled circles) food measured during an 8-week period. Wet-mash food was provided to mice starting from 2 weeks-of-age. Growth of male wild-type mice fed with dry pellets (filled triangles) is shown as a reference. Wild-type mice grew equally well with wetmash food (not shown). Feeding with wet-mash food significantly enhanced the growth of Gfra2 $2^{-/-}$mice compared with Gfra $2^{-/-}$mice fed with dry pellets. ${ }^{*} P<0.05$. 
Interestingly, most, if not all S100 $\beta$-positive enteric glial cells also express GFR $\alpha 2$. Moreover, we show that GFR $\alpha 2$ is expressed in nonmyelinating satellite cells that surround parasympathetic neurons in the ganglia, as well as in terminal Schwann cells in the pancreas and other exocrine glands (our unpublished data). This suggests that GFR $\alpha 2 /$ Ret-expressing neurons would be surrounded by GFR $\alpha 2$-expressing glial cells, which might have a role in target innervation, as suggested for GFR $\alpha 1$ (23).

Lack of GFR $\alpha 2$ results in impaired excitatory innervation of the gut muscle and in vivo motility. We show that during postnatal development, Nrtn mRNA expression appears to be concentrated in the circular muscle layer, as reported previously (24). We also found Nrtn in the circular muscle in adults, albeit at low levels. Thus, NRTN would be able to support those enteric neurons expressing GFR $\alpha 2 /$ Ret that innervate the intestinal muscles. Consistent with this idea, the number of SP-containing neurons in the myenteric plexus was reduced by about $35 \%$, which is consistent with the slightly reduced density of myenteric neurons in GFR $\alpha 2$-deficient mice (8). We also demonstrate that lack of GFR $\alpha 2$ signaling results in reduction in the density of SP fiber bundles at the level of the myenteric ganglion cell bodies in the small intestine. These fibers most likely correspond to the tertiary component of the myenteric plexus located against the inner face of the longitudinal muscle, which is suggested to be a major site for neurotransmission to the longitudinal muscle in the guinea pig (25). In contrast, SP fibers were not significantly affected in another layer of the myenteric plexus, the dmp, located on the border between the innermost circular layer and the submucosa (17). This would imply different mechanisms (e.g., another type of trophic factor) for controlling the density of SP-containing fibers in the different layers. For example, many enteric neurons (26) and glia (our unpublished data) also express GFR $\alpha 1$ after birth. Thus, a partially overlapping GDNF/GFR $\alpha 1$ signaling might explain the limited enteric innervation defect in Gfra2 ${ }^{-/-}$mice. The fact that GFR $\alpha 2$ is expressed similarly in these two layers (in fibers and/or glia) suggests that the preferential dependence of the tertiary plexus innervation on GFR $\alpha 2$ cannot fully be explained by lack of GFR $\alpha 2$ in the dmp-projecting neurons or their associated glia. Although some $25 \%$ of SP neurons in myenteric ganglia are GFR $\alpha 2$ negative, it is not known if these project preferentially to the $\mathrm{dmp}$ layer. Taken together, our results suggest that a specific subset of SP neurons is affected by the knockout, but the mechanism that account for the lesion of this particular cell group remains unclear. Additional deficits may exist in these mice in enteric neurons that we have not studied, for example, in the submucosal plexus.

Furthermore, we show here that GFR $\alpha 2$-deficient mice have impaired in vivo small bowel motility. In vitro, the small intestine in these animals has impaired contractility and rhythm (8). The excitatory innerva- tion of the intestinal muscles is crucial for normal propulsion (11). We suggest that at least part of the impaired in vivo motility is due to the impaired SP-positive innervation of the longitudinal and circular muscles. In addition, impaired neurotransmitter release without loss of innervation, as reported in NRTN-deficient mice (7), may contribute to the dysmotility in GFR $\alpha 2$-deficient mice.

Pancreas innervation deficit and its effect on exocrine function. Hormonal and neural reflexes that mediate foodinduced pancreatic enzyme secretion converge via the vagus nerve that activates postganglionic parasympathetic neurons in the pancreas $(9,10)$. In addition to the vagal efferent input, the intrapancreatic ganglion neurons receive direct sensory input from the stomach and duodenum (27), but the physiological role of the direct enteropancreatic pathway in the pancreas secretion is unknown. The intrapancreatic neurons relay the neural input to the pancreatic secretory cells but are also interconnected (28).

Knockout of GFR $\alpha 2$ resulted in a severe (>80\%) deficit of pancreatic parasympathetic neurons with an almost complete lack of intrinsic cholinergic innervation and a lack of vagally stimulated secretion of pancreatic zymogens. This is consistent with the established role of intrapancreatic neurons mediating food stimulus-induced pancreatic exocrine secretion. We demonstrate by measuring stool fat that the Gfra2 ${ }^{-/-}$ mice have malabsorption, most likely due to pancreatic insufficiency. The lack of overt steatorrhea suggests that the overall pancreas insufficiency is limited (relatively mild), presumably because of intact (or slightly elevated) basal pancreas enzyme secretion.

We show that the profound deficit of intrapancreatic neurons in GFR $\alpha 2$ knockout mice occurs mostly before P4. Similarly, submandibular parasympathetic ganglia require NRTN/GFR $\alpha 2$ signaling before birth to achieve the proper number of neurons $(5,6)$. Whether the reduced number of submandibular and intrapancreatic ganglion neurons in GFR $\alpha 2$-deficient mice reflects defect in migration, proliferation, or survival is subject of future studies. Vagal crest-derived cells colonize the duodenum by embryonic day 11 (E11) in rats, but do not seem to migrate into the pancreatic bud until E13 (29). The mechanisms that initially prevent and then mediate the postulated secondary migration of the precursors from bowel to pancreas are unknown. Enteric and some cranial parasympathetic precursors need GDNF during early development for migration and/or proliferation (3, 30-32). GDNF is expressed in the pancreatic rudiment (Vassilis Pachnis, personal communication) and could thus act as a possible enteropancreatic chemoattractant during the presumed colonization time (between E10-E13 in the mouse). The reported expression of NRTN mRNA in mouse pancreas between embryonic days E14 and E18 (18) would be consistent with a role for NRTN/GFR $\alpha 2$ in enteropancreatic precursor proliferation and later migration. Alternatively, NRTN may be 
crucial for commitment to the parasympathetic neuronal lineage, in which case GFR $\alpha 2$ deficiency might result in altered differentiation of these neurons along another neuronal lineage. Finally, NRTN/GFR $\alpha 2$ signaling may be needed in precursor or neuron survival. Whatever the mechanism, as pancreatic ganglia develop from crest-derived cells that are thought to migrate from the gut (29), there could be a common lineage of pancreatic and a subset of enteric neurons that is reflected in a shared GFR $\alpha 2$ dependence.

The Gfra $2^{-/-}$mice may provide a useful model to use to study the role of pancreatic innervation in the physiology of exocrine and endocrine pancreatic secretions. The cholinergic innervation of pancreatic islets appeared less affected compared with the exocrine part in Gfra $2^{-/-}$mice, suggesting that the parasympathetic innervation of endocrine tissue is predominantly derived from the subpopulation of pancreatic neurons that do not express GFR $\alpha 2$ and were still observed in the knockouts. It would also be of interest to determine pancreatic function in mice deficient in the M3 muscarinic receptor, the major cholinergic postganglionic receptor in the pancreas, which shows postweaning growth impairment similar to Gfra $2^{-/-}$mice $(33,34)$.

Partially rescued growth by wet-mash feeding suggests impaired saliva secretion. Physiological food-induced saliva secretion is largely mediated by the parasympathetic neurons; their stimulation usually evokes watery, low-protein secretion (35). In Gfra2 $2^{-/}$mice, the parasympathetic innervation is virtually missing in the sublingual gland and markedly reduced in the parotid gland (5), suggesting that food-induced saliva secretion could be compromised. Consistent with this hypothesis, we found that water intake of $\mathrm{G} f r a 2^{-/-}$mice was increased compared with wild-type mice. Moreover, if fed with wet-mash food, the knockout mice gained weight faster than if fed with dry pellets, but still significantly slower compared with controls. The growthpromoting ability of hydrated food, however, was only partial, suggesting that other defects in addition to salivary gland dysfunction contribute to poor growth.

Several mechanisms contribute to malnutrition in GFR $\alpha 2$ knockout mice. Based on the results of our study, we suggest that impaired secretion of pancreatic enzymes and saliva leads to poor digestion of food and malabsorption that contributes to the malnutrition and growth retardation in the Gfra2 $/-$ mice. The role of the small intestine dysmotility to the growth retardation is less clear because a slower transit could actually promote growth by giving more time for nutrient digestion and absorption. The Gfra2 $2^{-/-}$mice show normal locomotor activity and body temperature, whereas their BMR is elevated. The decreased fat content in these mice indicates higher muscle-to-fat ratio, leading to increased BMR, since fat is less metabolically active than muscle. The increased metabolic rate may also account for the animals' increased feeding per unit of body mass. Since serum glucose levels in fed Gfra2 $2^{-/}$mice are normal, hyperglycemia with urinary glucose loss cannot explain their impaired growth. Because regulation of endocrine pancreas secretion is influenced by parasympathetic stimulation (36), however, further studies on glucose metabolism are undertaken.

Energy homeostasis and food intake is under control by regulatory centers in the hypothalamus that express GDNF family receptors, including Gfra2 (37). The slightly increased food consumption of $\mathrm{Gfra}^{-/-}$mice, however, indicates that reduced appetite does not contribute to the poor growth; instead, the mutant animals might try to compensate their malnutrition by increasing food and water intake. This also suggests that, despite apparent salivary gland dysfunction, the mice may not have severe problems in swallowing.

It remains unclear why mice lacking NRTN (7), the preferred GFR $\alpha 2$ ligand, do not show a clear growthretarded phenotype as do the Gfra $2^{-/-}$mice (8). This suggests the possibility that other ligands, such as GDNF, compensate for the absence of NRTN. GDNF is able to signal via GFR $\alpha 2$ in vitro (1-4). So far, however, no clear anatomical or functional differences between the GFR $\alpha 2$ - and NRTN-deficient animals have been described that could explain their growth difference. This issue is being addressed in an ongoing study that compares the growth of these mice under the same genetic background and diet.

In conclusion, GFR $\alpha 2$-Ret signaling is needed for parasympathetic innervation including the exocrine pancreas and for SP-containing enteric innervation in the small intestine. The combined results of impaired secretion of the salivary gland and exocrine pancreas and intestinal dysmotility contribute to the growth retardation in the $\mathrm{Gfra}^{-/-}$mice.

\section{Acknowledgments}

We thank Mart Saarma for suggestions and Kaija Berg, Eila Kujamäki, and Riitta Kauppinen for technical assistance. This study was supported by grants from the Academy of Finland and the Sigrid Juselius Foundation (to M.S. Airaksinen).

1. Manié, S., Santoro, M., Fusco, A., and Billaud, M. 2001. The RET receptor: function in development and dysfunction in congenital malformation. Trends Genet. 17:580-589.

2. Takahashi, M. 2001. The GDNF/RET signaling pathway and human diseases. Cytokine Growth Factor Rev. 12:361-373.

3. Airaksinen, M.S., and Saarma, M. 2002. The GDNF family: signalling, biological functions and therapeutic value. Nat. Rev. Neurosci. 3:383-394.

4. Baloh, R.H., Enomoto, H., Johnson, E.M.J., and Milbrandt, J. 2000. The GDNF family ligands and receptors - implications for neural development. Curr. Opin. Neurobiol. 10:103-110.

5. Rossi, J., Tomac, A., Saarma, M., and Airaksinen, M.S. 2000. Distinct roles for GFR $\alpha 1$ and GFR $\alpha 2$ signalling in different cranial parasympathetic ganglia in vivo. Eur. J. Neurosci. 12:3944-3952.

6. Enomoto, H., Heuckeroth, R.O., Golden, J.P., Johnson, E.M., and Milbrandt, J. 2000. Development of cranial parasympathetic ganglia requires sequential actions of GDNF and neurturin. Development. 127:4877-4889.

7. Heuckeroth, R.O., et al. 1999. Gene targeting reveals a critical role for neurturin in the development and maintenance of enteric, sensory, and parasympathetic neurons. Neuron. 22:253-263.

8. Rossi, J., et al. 1999. Retarded growth and deficits in the enteric and parasympathetic nervous system in mice lacking GFR $\alpha 2$, a functional neurturin receptor. Neuron. 22:243-252.

9. Owyang, C. 1996. Physiological mechanisms of cholecystokinin action on pancreatic secretion. Am. J. Physiol. 271:G1-G7. 
10. Rogers, R.C., McTigue, D.M., and Hermann, G.E. 1996. Vagal control of digestion: modulation by central neural and peripheral endocrine factors. Neurosci. Biobehav. Rev. 20:57-66.

11. Sang, Q., and Young, H.M. 1998. The identification and chemical coding of cholinergic neurons in the small and large intestine of the mouse. Anat. Rec. 251:185-199.

12. Bianchi, G., et al. 1983. Morphine tissue levels and reduction of gastrointestinal transit in rats. Correlation supports primary action site in the gut. Gastroenterology. 85:852-858.

13. Miyasaka, K., et al. 1999. Disruption of cholecystokinin (CCK)-B receptor gene did not modify bile or pancreatic secretion or pancreatic growth: a study in CCK-B receptor gene knockout mice. Pancreas. 19:114-118.

14. Winn-Deen, E.S., David, H., Sigler, G., and Chavez, R. 1988. Development of a direct assay for alpha-amylase. Clin. Chem. 34:2005-2008.

15. Jeejeebhoy, K.N., Ahmad, S., and Kozak, G. 1970. Determination of fecal fats containing both medium and long chain triglycerides and fatty acids. Clin. Biochem. 3:157-163.

16. Dinulescu, D.M., et al. 1998. Mahogany (mg) stimulates feeding and increases basal metabolic rate independent of its suppression of agouti. Proc. Natl. Acad. Sci. U. S. A. 95:12707-12712.

17. Wilson, A.J., Llewellyn-Smith, I.J., Furness, J.B., and Costa, M. 1987. The source of the nerve fibres forming the deep muscular and circular muscle plexuses in the small intestine of the guinea-pig. Cell Tissue Res. 247:497-504.

18. Golden, J.P., DeMaro, J.A., Osborne, P.A., Milbrandt, J., and Johnson, E.M., Jr. 1999. Expression of neurturin, GDNF, and GDNF family-receptor mRNA in the developing and mature mouse. Exp. Neurol. 158:504-528.

19. Ekblad, E., Alm, P., and Sundler, F. 1994. Distribution, origin and projections of nitric oxide synthase-containing neurons in gut and pancreas. Neuroscience. 63:233-248.

20. Havel, P.J., and Taborsky, G.J.J. 1989. The contribution of the autonomic nervous system to changes of glucagon and insulin secretion during hypoglycemic stress. Endocr. Rev. 10:332-350.

21. Li, Y., Jiang, Y.C., and Owyang, C. 1998. Central CGRP inhibits pancreatic enzyme secretion by modulation of vagal parasympathetic outflow. Am. J. Physiol. 275:G957-G963.

22. Rogers, P., and Webb, G.P. 1980. Estimation of body fat in normal and obese mice. Br. J. Nutr. 43:83-86.

23. Ledda, F., Paratcha, G., and Ibanez, C.F. 2002. Target-derived GFR $\alpha 1$ as an attractive guidance signal for developing sensory and sympathetic axons via activation of Cdk5. Neuron. 36:387-401.

24. Widenfalk, J., et al. 1997. Neurturin and glial cell line-derived neu- rotrophic factor receptor-beta (GDNFR- $\beta$ ), novel proteins related to GDNF and GDNFR- $\alpha$ with specific cellular patterns of expression suggesting roles in the developing and adult nervous system and in peripheral organs. J. Neurosci. 17:8506-8519.

25. Llewellyn-Smith, I.J., Costa, M., Furness, J.B., and Bornstein, J.C. 1993. Structure of the tertiary component of the myenteric plexus in the guinea-pig small intestine. Cell Tissue Res. 272:509-516.

26. Chalazonitis, A., Rothman, T.P., Chen, J., and Gershon, M.D. 1998. Agedependent differences in the effects of GDNF and NT-3 on the development of neurons and glia from neural crest-derived precursors immunoselected from the fetal rat gut: expression of GFR $\alpha-1$ in vitro and in vivo. Dev. Biol. 204:385-406.

27. Kirchgessner, A.L., and Gershon, M.D. 1990. Innervation of the pancreas by neurons in the gut. J. Neurosci. 10:1626-1642.

28. Liu, M.T., and Kirchgessner, A.L. 1997. Guinea pig pancreatic neurons: morphology, neurochemistry, electrical properties, and response to 5-HT. Am. J. Physiol. 273:G1273-G1289.

29. Kirchgessner, A.L., Adlersberg, M.A., and Gershon, M.D. 1992. Colonization of the developing pancreas by neural precursors from the bowel. Dev. Dyn. 194:142-154.

30. Young, H.M., et al. 2001. GDNF is a chemoattractant for enteric neural cells. Dev. Biol. 229:503-516.

31. Natarajan, D., Marcos-Gutierrez, C., Pachnis, V., and de Graaff, E. 2002. Requirement of signalling by receptor tyrosine kinase RET for the directed migration of enteric nervous system progenitor cells during mammalian embryogenesis. Development. 129:5151-5160.

32. Gianino, S., Grider, J.R., Cresswell, J., Enomoto, H., and Heuckeroth, R.O. 2003. GDNF availability determines enteric neuron number by controlling precursor proliferation. Development. 130:2187-2198.

33. Matsui, M., et al. 2000. Multiple functional defects in peripheral autonomic organs in mice lacking muscarinic acetylcholine receptor gene for the M3 subtype. Proc. Natl. Acad. Sci. U. S. A. 97:9579-9584.

34. Yamada, M., et al. 2001. Mice lacking the M3 muscarinic acetylcholine receptor are hypophagic and lean. Nature. 410:207-212.

35. Garrett, J.R. 1987. The proper role of nerves in salivary secretion: a review. J. Dent. Res. 66:387-397.

36. Ahren, B. 2000. Autonomic regulation of islet hormone secretion implications for health and disease. Diabetologia. 43:393-410.

37. Golden, J.P., et al. 1998. Expression of neurturin, GDNF, and their receptors in the adult mouse CNS. J. Comp. Neurol. 398:139-150.

38. West, G.B., Brown, J.H., and Enquist, B.J. 1999. The fourth dimension of life: fractal geometry and allometric scaling of organisms. Science. 284:1677-1679. 